Flores Pacheco, M. \& Larios León, S. (2014). Aportes para el estudio del territorio: Los Nahuas de Zongolica Veracruz. Collectivus, Revista de Ciencias Sociales, 1, (1), 70-93. Julio - Diciembre. ISSN: 2382-4018

Recibido: 10/10/2013

Aceptado versión definitiva: 10/01/2014

\title{
APORTES PARA EL ESTUDIO DEL TERRITORIO: LOS NAHUAS DE ZONGOLICA VERACRUZ ${ }^{1}$
}

\author{
MARTHA INÉS FLORES PACHECO² / SOFÍA LARIOS LEÓN ${ }^{3}$ \\ Instituto de Antropología / Universidad Veracruzana, Av. Xalapa \#310, colonia \\ Progreso Macuiltepetl, CP. 91130, Xalapa, Veracruz, México \\ martflores@uv.mx; slarios@uv.mx
}

\section{RESUMEN}

El estudio sobre el tema del territorio indígena es muy amplio y puede analizarse desde diferentes perspectivas teórico metodológicas, no obstante, este artículo lo aborda principalmente desde el enfoque de la antropología y el trabajo etnográfico. La región de estudio es la zona nahua de Zongolica donde se está realizando el proyecto de investigación intitulado: Políticas sociales y Pueblos Indígenas; turismo y patrimonio histórico, cultural y natural de la Región de las Montañas, Veracruz. En el presente artículo se exponen los avances de la revisión teórica sobre la territorialidad indígena y su vinculación con la cosmovisión indígena, los derechos indígenas y el patrimonio histórico cultural y natural, territorialidad que está siendo violentada por otros grupos sociales y acciones de desarrollo promovidas por el Estado Mexicano.

Palabras clave: Territorio indígena, Geografía simbólica, patrimonio cultural, proyectos turísticos.

\footnotetext{
${ }^{1}$ El presente artículo se enmarca en el proyecto de investigación sobre Políticas Sociales y Pueblos Indígenas: Turismo y Patrimonio Histórico, Cultural y Natural de la Región de las Montañas de Veracruz.

${ }^{2}$ Doctora en Ciencias Antropológicas por la Universidad Autónoma Metropolitana Iztapalapa.

${ }^{3}$ Candidato a Doctor en Historia Contemporánea por la Universidad del País Vasco.
} 
Flores Pacheco, M. \& Larios León, S. (2014). Aportes para el estudio del territorio: Los Nahuas de Zongolica Veracruz. Collectivus, Revista de Ciencias Sociales, 1, (1), 70-93. Julio - Diciembre. ISSN: 2382-4018

CONTRIBUTIONS TO THE STUDY OF THE TERRITORY: THE NAHUAS OF ZONGOLICA, VERACRUZ

\section{ABSTRACT}

The study on the issue of the indigenous territory is very broad and can be analysed from different methodological theoretical perspectives, however, this study tackles it from the focus of anthropology and ethnographic work. The region of study where this research project is underway is the nahua area of Zongolica and is entitled: Social policies and indigenous peoples; tourism and historic, cultural and natural heritage of the Region of the Mountains, Veracruz. This paper presents advances in the theoretical review on indigenous territory and its bonding to the indigenous worldview, indigenous rights and the cultural and natural heritage, territoriality which is being raped by other social groups and development actions promoted by the Mexican State.

Key descriptors: indigenous territory, symbolic geography, cultural heritage, tourism projects.

\section{Introducción}

Hablar del concepto de territorio y de territorialidad en regiones indígenas de México es importante, por ser el espacio donde históricamente han vivido estos pueblos y lo han rodeado de significados. En estos territorios se encuentra su pasado, su presente y su futuro. La forma en que los pueblos indígenas de México han conservado su territorio presenta algunas similitudes, sobre todo los que provienen de un origen mesoamericano. Encontrándonos con algunos estudios sobre la concepción indígena de territorio desde la época prehispánica, el siglo XVI y hasta nuestros días.

El interés por estudiar el tema del territorio en la región nahua de Zongolica, responde a la necesidad de profundizar en la comprensión de la forma en que los pueblos indígenas utilizan su territorio. De cómo los nombran y de cómo se dan diferentes rituales para conservarlo y a su vez, cómo sus 
Flores Pacheco, M. \& Larios León, S. (2014). Aportes para el estudio del territorio: Los Nahuas de Zongolica Veracruz. Collectivus, Revista de Ciencias Sociales, 1, (1), 70-93. Julio - Diciembre. ISSN: 2382-4018

territorios son actualmente vistos como lugares de atracción turística o de proyectos de desarrollo provocando una pérdida irreparable de su identidad.

México cuenta con más de 62 grupos etnolingüísticos que se encuentran a lo largo y ancho del país, cada uno de ellos cuenta actualmente con territorios diferenciados de la población mestiza y en algunos casos conviven con otros grupos. Por lo tanto, México es un país multilingüista, multiétnico, multicultural y multirracial.

Sobre este tema, realizamos con otros pueblos indígenas desde hace varios años, siendo los trabajos más importantes los siguientes:

Con el pueblo indígena Cora, que se encuentran ubicado en la región de la Sierra Madre Oriental, es importante la relación entre los relatos míticos coras (mitos de origen) y la geografía de la Sierra Madre Occidental (geografía mítica). Cada familia extensa Cora habita un paraje en época de lluvia llamado "rancho de agua". Estos parajes tienen diferentes nombres de lugar. Los Coras consideran que en su territorio existen sitios donde los hombres y los dioses vivieron diferentes acontecimientos en el pasado histórico y mítico. Estos lugares se encuentran en los mitos o "historias antiguas" y en los relatos 0 "historias de oídas". La mayoría de los relatos se relacionan con el territorio mítico, histórico y ancestral, de este modo estos lugares tienen el mismo nombre y significado en la actualidad (Flores, 1991, 2007).

En la Huasteca potosina, con el propósito de contribuir en el conocimiento de la concepción que tienen pueblos Nahuas y Teenek sobre su territorialidad. Al mismo tiempo que entender cómo se han apropiado de ella sus habitantes a partir de la lengua, la geografía, la etnohistoria y la ecología; se abarcaron tres grandes contenidos: 1.- Se reconstruyó la historia del territorio de la Huasteca, tomando en consideración la tenencia de la tierra, la organización social, política y territorial, así como las movilizaciones sociales que los habitantes han tenido en torno al uso y posesión a la tierra; 2.- se conocieron los significados toponímicos, empleados por los habitantes Nahuas y Teenek, para construir y delimitar sus territorios reales y simbólicos a través de la etnografía mítica, mismos que se expresan en la existencia y uso de los lugares sagrados y, 3.- se conoció y analizó los conflictos interétnicos actuales 
Flores Pacheco, M. \& Larios León, S. (2014). Aportes para el estudio del territorio: Los Nahuas de Zongolica Veracruz. Collectivus, Revista de Ciencias Sociales, 1, (1), 70-93. Julio - Diciembre. ISSN: 2382-4018

vigentes en los territorios de los Nahuas y Teenek y que se expresan en torno a los lugares sagrados (Flores, 2007).

En la Montaña de Guerrero, con el pueblo indígena Xabume'phaa o Tlapaneco, se realizó un estudio sobre el territorio indígena, a partir de la revisión de la historia, de su proceso de identidad y de los elementos culturales que lo identifican como grupo etnolingüístico. Se encontró que en este territorio, en el que interactúan distintos grupos etnolingüísticos, existen añejos problemas por los límites territoriales. Ya sea por la falta de precisión de los colindantes (los límites y mojoneras son movibles pues se trata de veredas, caminos, piedras, cerros, árboles, entre otros, ocasionando problemas que han enfrentado a las comunidades), por deficiencias legales en los expedientes, la lejanía geográfica y por la indeterminación de los linderos de los municipios, las comunidades agrarias, localidades, cuadrillas y ranchos (Flores, 2010).

Para el caso de la región de Zongolica, se puede agregar que la comarca presenta una larga trayectoria de conflictos agrarios en torno a la tenencia de la tierra. La complejidad del problema tiene que ver con una concepción particular de territorialidad étnica. El compartir los símbolos identitarios y los geosímbolos, ha llevado consigo a la disputa por el espacio geográfico y el mantenimiento de los límites étnicos del grupo original Nahua y su disputa histórica con los mestizos que se asentaron en la región desde hace siglos.

El estudio sobre el territorio Nahua en Zongolica, se enmarca en el proyecto de investigación: Políticas sociales y pueblos indígenas: Turismo y patrimonio histórico, cultural y natural de la Región de las Montañas, Veracruz; ubicada en una de la zonas centrales del estado, en la franja donde existe una importante diversidad cultural y étnica. La sierra de Zongolica es parte de la Sierra Madre Oriental, cuenta con un territorio aproximado de $1714.9 \mathrm{~km}^{2}$, y comprende los municipios de Astacinga, Atlahuilco, Magdalena, Mixtla de Altamirano, Los Reyes, San Andrés Tenejapan, Soledad Atzompa, Tehuipango, Tequila, Texhuacan, Tlaquilpa, Xoxocotla y Zongolica y junto a ellas Coetzala. Limita al sur con la Sierra Mazateca de Oaxaca, al este con la 
Flores Pacheco, M. \& Larios León, S. (2014). Aportes para el estudio del territorio: Los Nahuas de Zongolica Veracruz. Collectivus, Revista de Ciencias Sociales, 1, (1), 70-93. Julio - Diciembre. ISSN: 2382-4018

Llanura Costera del Golfo; al oeste con la vertiente occidental de la misma Sierra; y al norte con el Pico de Orizaba y el Cañón de Río Blanco.

El propósito de la investigación es analizar la concepción de territorialidad étnica y patrimonio histórico cultural a partir de fuentes directas e indirectas. Identificando los elementos culturales de la identidad indígena y los efectos que están teniendo los proyectos turísticos en el proceso de reproducción cultural.

Con este estudio se busca aportar elementos que coadyuven a una mayor comprensión sobre la territorialidad indígena y los cambios y afectaciones que se dan con la aplicación de políticas y programas de desarrollo que inciden en su territorio. Los cuales terminan mermando o disminuyendo aspectos importantes de la identidad indígena como son: Los sistemas y normas internas y de comportamiento, usos y manejo de los recursos naturales, formas de vida, procesos organizativos, distribución espacial de la comunidad y la práctica de los usos y costumbres en relación a la tenencia de la tierra; así como costumbres, territorios sagrados y fiestas. La complejidad que esto implica, hace necesario los estudios puntuales para profundizar en el conocimiento de la problemática que enfrentan.

En este espacio se presentan algunas de las reflexiones teórico metodológicas sobre el tema considerando variables multifactoriales, interdisciplinarias y multidisciplinarias. El territorio se aborda como el eje articulador de la cultura, patrimonio histórico (cultural y natural), turismo y derechos indígenas, dimensiones relacionadas entre sí que no pueden disociarse para comprender la situación indígena. El turismo es una actividad que genera impactos sobre el territorio en las regiones indígenas, moldea relaciones socioeconómicas locales e incide en los aspectos simbólicos culturales que constituyen el mismo territorio. El turismo y las políticas sociales del Estado generan transformaciones que pueden amenazar el territorio, el ambiente, las costumbres y las tradiciones de la población local, esto es, el patrimonio histórico, cultural y natural indígena. 
Flores Pacheco, M. \& Larios León, S. (2014). Aportes para el estudio del territorio: Los Nahuas de Zongolica Veracruz. Collectivus, Revista de Ciencias Sociales, 1, (1), 70-93. Julio - Diciembre. ISSN: 2382-4018

\section{El estado de la cuestión: Conceptos, temas y perspectivas}

El concepto de territorio nos remite a la noción de espacio. Espacio y territorio son significaciones que permanecen relacionados de distinta forma en determinadas disciplinas, ambos conceptos lejos de ser entidades por sí mismas, no le pertenecen a ninguna disciplina de manera única y exclusiva. Los conceptos de espacio y territorio han estado trabajados, tanto desde las ciencias naturales con sus métodos y técnicas (geografía y astronomía), humanistas, filosóficas y exactas (ecología y etología), así como por las ciencias sociales (economía o ecología política), como también en algunas escuelas teóricas y corrientes antropológicas. Igualmente, se escucha hablar de "territorio nacional" desde las políticas gubernamentales y "territorio indígena" desde la antropología aplicada. Estas disciplinas tratan sobre el espacio y/o el territorio. Por tanto, la discusión sobre la relación entre espacio y territorio puede ser interminable.

Desde la óptica del tiempo, el espacio sirve de contenedor a múltiples actividades. El territorio es espacio construido por y en el tiempo. De esta manera, cualquier espacio habitado por el hombre es producto del tiempo de la naturaleza, del tiempo de los humanos, de las distintas formas de organización y de la concepción cosmogónica del tiempo.

En esta noción se incluye una dimensión política del territorio. Es decir, en la relación con lo ambiental y con su alteridad el hombre se apropia de espacios, determina rutas, hace señalamientos. Establece un dominio en un área geográfica específica. Se construye un sentido de la identidad espacial que guarda exclusividad y supone división en la interacción humana. Esto redunda en conflicto por el espacio limitado. El territorio es así más que el espacio; son varios los territorios posibles en un espacio geográfico común.

Una perspectiva para abordar la noción de territorio es la que ofrece Gilberto Giménez, que señala que "uno de los beneficios invaluables que podríamos esperar de este acercamiento transdisciplinario podría ser la 
Flores Pacheco, M. \& Larios León, S. (2014). Aportes para el estudio del territorio: Los Nahuas de Zongolica Veracruz. Collectivus, Revista de Ciencias Sociales, 1, (1), 70-93. Julio - Diciembre. ISSN: 2382-4018

recuperación del sentido del contexto espacio-temporal o geohistórico como matriz indisociable de los hechos sociales que constituyen nuestro objeto de estudio" (Giménez, 2005, p. 8).

Giménez propone recuperar de la nueva geografía el concepto de territorio o territorialidad. Éste permite entender las identidades sociales territorializadas, como las de los pueblos indígenas y encuadrar adecuadamente los fenómenos de arraigo, del apego y del sentimiento de pertenencia socio-territorial. El territorio como espacio apropiado, es de naturaleza multiescalar, es decir, puede ser aprehendido en diferentes niveles de la escala geográfica: Local, regional, nacional e internacional. También el territorio se relaciona con el paisaje como instancia privilegiada de la percepción territorial, en la que los actores invierten en forma entremezclada su afectividad, su imaginario y su aprendizaje socio-cultural. Incorpora la dimensión simbólica o cultural del territorio que se interesa por la interpretación simbólica que los grupos y las clases sociales hacen de su entorno, las justificaciones estéticas o ideológicas que proponen y el impacto de las representaciones sociales sobre la modelación del paisaje. Todos estos conceptos, para Giménez (2005), permiten encuadrar fenómenos sociales que tienen que ver con la territorialidad.

\subsection{El Territorio y cultura / concepción simbólica}

El territorio puede ser analizado desde la perspectiva de lo simbólico. Es decir, desde la concepción sagrada que tienen los pueblos indígenas actuales sobre el espacio en el que viven y cómo en el conviven diferentes elementos en común que se presentan en la tradición de los pueblos originarios de México y, en particular, de los nahuas de la sierra de Zongolica.

Giménez y Héau (2007, p. 8), proponen conceptos claves de la teoría del territorio: 1.- El territorio como apropiación del espacio; 2.- el paisaje como condensación metonímica del territorio; 3.- los geo- símbolos como referentes de la identidad y 4.- el territorio como representación; al igual que otros conceptos delimitados simbólicamente por el territorio. La relación del territorio 
Flores Pacheco, M. \& Larios León, S. (2014). Aportes para el estudio del territorio: Los Nahuas de Zongolica Veracruz. Collectivus, Revista de Ciencias Sociales, 1, (1), 70-93. Julio - Diciembre. ISSN: 2382-4018

con la geografía, la posición del hombre en el universo, el origen del hombre, cosmos y territorio, la relación con los pasados, los seres que viven en él, por mencionar algunos.

El territorio y la territorialidad se miran desde la antropología:

(...) por un lado, como una construcción cultural donde tienen lugar las prácticas sociales con intereses distintos, con percepciones, valoraciones y actitudes territoriales diferentes, que generan relaciones de complementación, de reciprocidad, pero también de confrontación. Dicha construcción es susceptible de cambios según las épocas y las dinámicas sociales. $\mathrm{Y}$ por el otro, como portador de una doble naturaleza. Una que lo convierte en un tema para el estudio de una sociedad, en la que el territorio es sólo un medio. $Y$ otra naturaleza de tipo más cosmogeográfico, en la que el territorio cobra cuerpo como objeto mismo de estudio.

Por lo que asumimos que no existe un territorio en sí, sólo existe un territorio para alguien que puede ser un actor social, tanto individual como colectivo, que lo influye desde la planeación y el ordenamiento territorial, hasta el decoro del hogar o el acceso a los recursos. Además, el uso social del territorio no se puede tomar en abstracto: Se concreta en dominios culturales tales que el parentesco, la economía, la salud, la política, la religión, entre otros.... Lo que significa que las disposiciones territoriales son garantes de la identidad social y cultural, y que aseguran su reproducción. (Nantes Cruz, 2011, 2012).

Por lo tanto, el análisis cultural y simbólico del territorio, tiene que tomar en cuenta los conceptos que proponen Giménez y Héau (2007), así como los que formulan Barabas (2004), López Austín (1994), Velasco Toro (2002), entre otros, debido a que dan herramientas para acercarse a la compleja relación que los pueblos indígenas establecen con su entorno. En la actualidad, la mayoría de los pueblos indígenas mantienen una relación estrecha con el universo y el territorio, la geografía y el inframundo. 
Flores Pacheco, M. \& Larios León, S. (2014). Aportes para el estudio del territorio: Los Nahuas de Zongolica Veracruz. Collectivus, Revista de Ciencias Sociales, 1, (1), 70-93. Julio - Diciembre. ISSN: 2382-4018

Aguirre Beltrán (1986) y Velasco Toro (2002) mencionan que existe una correspondencia entre el hombre, las deidades y el territorio (naturaleza), desde lo histórico, cultural y social, por medio de por lo menos cinco elementos: 1) Concepción sagrada del mundo; 2) Imagen territorial y sus límites, 3) Reconstitución étnica a partir de la organización familiar; 4) Memoria histórica y 5) La soberanía comunitaria en la que reside la responsabilidad del territorio cedido por los dioses al hombre, por lo que tiene la obligación de renovar el vínculo deidades-hombres. Esta concepción del mundo se encuentra vinculada con la identidad y la territorialidad de sus habitantes, del mismo modo que con algunos rituales que se practican actualmente.

La geografía simbólica proporciona un enfoque integrador del etnoterritorio que recupera categorías y conocimientos culturales propios y muestra los lugares sagrados o étnicos, los cuales marcan centros y fronteras. La concepción de territorio como espacio culturalmente construido por la sociedad, se refiere a que se busca identificar las pautas seguidas por los grupos etnolingüísticos para la construcción de territorios y lugares en ámbitos locales y globales. Es decir interpretar los significados sociales y culturales que tiene el territorio, en sus diversos niveles, para los indígenas que lo ocupan históricamente. La dimensión espacial (medioambiente, la tierra) se refiere a la base física sobre la que un pueblo se asienta.

El territorio es una construcción social, resultado de diversas formas de apropiación del espacio del que participan diversos actores. Estas múltiples apropiaciones se nutren de la cultura, de la memoria y las experiencias sociales surgidas de los conflictos territoriales o agrarios, así como de los procesos históricos resultados de las relaciones con el estado-nacional. Por tanto, la idea de territorio aunque tiene como referente o soporte principal la geografía, el ambiente físico y los recursos naturales, es más bien un producto social y cultural. En la construcción social del territorio participan un conjunto de actores que despliegan distintas territorialidades, esto es, formas específicas de apropiación del territorio, que a veces se encuentran en posiciones antagónicas. 
Flores Pacheco, M. \& Larios León, S. (2014). Aportes para el estudio del territorio: Los Nahuas de Zongolica Veracruz. Collectivus, Revista de Ciencias Sociales, 1, (1), 70-93. Julio - Diciembre. ISSN: 2382-4018

Barabas (2004) sostiene que las regionalizaciones fabricadas por el Estado tienen atributos y funciones impuestos por su lógica e intereses, que desconocen los criterios históricos, territoriales, culturales y étnicos, significativos para los indígenas. Las regiones, distritos, municipios y jurisdicciones agrarias resultantes de tal "omisión" han contribuido a fragmentar a los Pueblos Indígenas, a opacar el conocimiento local sobre el espacio compartido, a crear una falsa imagen de discontinuidad territorial y minoría; asimismo han conducido a conflictos por límites, a la desunión y a la pérdida de fuerza colectiva, muchas veces entre comunidades del mismo Pueblo.

\subsubsection{La concepción del mundo de los Nahuas de Zongolica}

El hombre Nahua, concibe que él reside en el centro del mundo o maxi mundo, y a partir de allí este se encuentra delimitado por los cerros sagrados y por el mundo celeste y el inframundo. Este mundo, además, se encuentra vinculado con diferentes divinidades que son las que sostienen a los hombres y éstos a su vez a ellas. Esta geografía simbólica contiene lugares que representan algún hecho social o cultural. El espacio no es estático, es flexible ya que en el interactúan naturaleza, dioses fundadores, lugares de origen, mitos, individuos, familia, comunidad, instituciones y antepasados de los nahuas actuales ${ }^{4}$.

De acuerdo a Rodríguez (2003) $)^{5}$, en la concepción territorial de los nahuas de la Sierra de Zongolica existe una metáfora cósmica de la oposición

\footnotetext{
4 "El espacio sagrado en la tradición etnocultural de Yaquis, Zoques y Chinantecos, se concibe relacionado con un ser, o seres supremos. (...) se observa un valor gnoseológico y etnocéntrico común: El carácter sagrado, cosmológico y multidimensional del espacio (Velasco Toro, 2002, p.144). "Las deidades de los Nahuas se ubican también en el seno de esta concepción bipartita del universo: Las deidades húmedas, frías, nocturnas que habitan en el subsuelo y las deidades terrestres solares y divinas que habitan en la superficie y en el espacio celeste..." (Rodríguez, 2003, pp. 237-238).

5 "El espacio regional constituye una unidad territorial significativa en las interacciones entre las localidades y el entorno extrarregional, representando una forma de intermediación que trasciende las divisiones intrarregionales. El manejo de este marco común de referencia constituye una instancia de identificación que se manifiesta en el uso de una misma lengua, un territorio compartido y una serie de elementos que configuran lo que podría llamarse una región cultural. Se conocen con precisión las rutas de acceso a cada pueblo, las devociones locales, los hábitos y productos específicos de cada una de ellas, los modismos idiomáticos, los
} 
Flores Pacheco, M. \& Larios León, S. (2014). Aportes para el estudio del territorio: Los Nahuas de Zongolica Veracruz. Collectivus, Revista de Ciencias Sociales, 1, (1), 70-93. Julio - Diciembre. ISSN: 2382-4018

arriba- abajo, arriba Tlaquitepatl (superficie terrestre) madre de los agricultores y abajo Tlalokan señor de la tierra y habitante del subsuelo, dueño de los animales y protector de la vegetación silvestre; ambos se encuentran en constante diálogo. Las cañadas húmedas y profundas de la tierra caliente constituyen un espacio incierto y peligroso y son donde se llevan las ofrendas el día de la Santa Cruz (petición de lluvias y bendición de los nacimientos de agua $)^{6}$.

En la religiosidad de los Nahuas de Zongolica (...) destaca la existencia de Tlalokan como un ámbito subterráneo de riqueza y abundancia que desemboca en las cuevas de las montañas y es morada de los Tlalokes, deidades relacionadas con las lluvias, los animales silvestres y la vegetación. (Rodríguez, 2003, p. 224).

Esta autora se basa en la obra de López Austin (1994), sobre la concepción dual de los pueblos mesoamericanos: arriba-abajo, cieloinframundo, este-oeste, por mencionar algunos:

(...) Por ello, el monte, las cuevas, los manantiales y caminos abandonados, los cementerios y los funerales son lugares peligrosos per se. Las cualidades asociadas a la parte femenina del universo son frías y hasta cierto punto malignas, mientras que lo masculino es caliente y vital. De esta clasificación primaria se deriva una serie de equivalencias que rigen la concepción del universo de los Nahuas. (Rodríguez, 2003, p. 235).

La autora agrega que el sistema de poblamiento es disperso basado en el parentesco, dividido en parajes, barrios, congregaciones, grupos de ojo de agua, linajes, grupos de descendencia, segmentos, subcomunidades 0

detalles de la indumentaria. "Esta visión de espacio regional esta signada por mitos de origen y lugares sagrados que posibilitan su aprehensión simbólica (...)” (Rodríguez, 2003, p. 34).

6 "Esta geografía simbólica mítica pone de relieve los contrastes ecológicos, la organización social del espacio y la idea de un devenir temporal regido por la sucesión de fiestas en honor de las divinidades..." (Rodríguez, 2003, p. 35). Retoma a Galanier (1990, pp. 42-43) quien menciona que los Nahuas desde la antigüedad tienen la idea de que todo espacio habitado mantiene correspondencia simbólica con un punto cardinal situado en un punto elevado (Rodríguez, 2003, p. 36). 
Flores Pacheco, M. \& Larios León, S. (2014). Aportes para el estudio del territorio: Los Nahuas de Zongolica Veracruz. Collectivus, Revista de Ciencias Sociales, 1, (1), 70-93. Julio - Diciembre. ISSN: 2382-4018

subdivisiones, departamentos, congregaciones y rancherías. Cada lugar tiene un topónimo, que tiene que ver con las características del sitio donde está ubicado el poblado. Estos topónimos destacan las subdivisiones internas para ubicar las unidades domesticas ${ }^{7}$ (Rodríguez, 2003, pp. 38-49).

Esta dimensión simbólica ha sido muy importante en el proceso histórico de conformación del territorio en la región de Zongolica y en la concepción de territorialidad étnica. A partir de los símbolos identitarios y geo símbolos que comparten estos pueblos.

En el espacio se van construyendo diferentes significados, prácticas, pertenencias y límites, en la medida que un pueblo vive allí, se sustenta de él y crea historia, sociedad y cultura en relación con ese medio ambiente. La falta de reconocimiento de esta concepción indígena ha llevado consigo a la disputa por el espacio geográfico y el mantenimiento de los límites étnicos del grupo original Nahua y un conflicto histórico con los mestizos que se asentaron en la región desde hace siglos.

\subsection{El Patrimonio histórico, cultural y natural étnico}

Otro tema importante que contienen los territorios indígenas es el patrimonio histórico-cultural, entendido como un legado de conocimientos explícitos e implícitos de un pueblo en torno a su identidad, organización social, concepción de vida, relación con la naturaleza y la sociedad. Este contiene diferentes especialidades, como el cultural, natural, paleontológico, arqueológico, histórico, lingüístico, social, por nombrar algunos y su estudio se ha dado desde diferentes campos de análisis.

El patrimonio cultural representa la riqueza apreciada de la cultura de un pueblo, constituida por bienes, valores, prácticas del carácter más diverso y originados en tiempos muy distintos. El concepto de patrimonio puede

\footnotetext{
7 Este sistema toponímico permite diferenciar cada uno de los niveles de adscripción residencial mediante un sistema de círculos concéntricos en la cual la casa o el espacio residencial es la unidad mínima. Lugar- paraje - congregación y municipio. Retoma a Sandstrom (1997, p. 22) en el aspecto de que este sistema de toponimia permite que parientes y no parientes se identifiquen con un mismo topónimo, el de su espacio residencial, permitiendo de este modo incorporar en una misma categoría de papeles sociales a los miembros de la casa (Rodríguez, 2003, p. 55).
} 
Flores Pacheco, M. \& Larios León, S. (2014). Aportes para el estudio del territorio: Los Nahuas de Zongolica Veracruz. Collectivus, Revista de Ciencias Sociales, 1, (1), 70-93. Julio - Diciembre. ISSN: 2382-4018

considerarse como un todo relacionado con su identidad. Los pueblos indígenas Nahuas plantean que dentro de su patrimonio se encuentran los lugares sagrados ${ }^{8}$.

El patrimonio contiene los elementos culturales que hace a los habitantes actuales poseedores de un patrimonio natural (conocimiento de la flora y la fauna), cultural (valores, conocimientos, memoria colectiva, apropiación de elementos culturales regionales), arqueológico (“montículos" lugar donde se encuentran los pasados, importancia de conservarlo), histórico (mapas, documentos, construcciones antiguas y libros), medicinal (aprovechamiento de plantas curativas regionales, uso de la magia, brujería y medicina tradicional), ecológico (preservación y conservación del medio ambiente), lingüístico (lengua, usos y educación), social (sistema de gobierno tradicional, elección de autoridades) ${ }^{9}$ y religioso (mitología, creencias, rituales a los lugares sagrados y respeto a los "dueños de la naturaleza"), como poseedores de una cultura milenaria y que continúa hasta nuestros días (Toledo, 1997, p. 131).

Culturalmente, los habitantes de esta región se han apropiado de los elementos de la naturaleza a lo largo de los siglos que llevan viviendo en la sierra. Conocen los recursos bióticos, energéticos o minerales, esto ocurre en una doble dimensión: Como apropiación material (producción de bienes) y como apropiación intelectual (producción de símbolos).

Se trata de los procesos mediante los cuales las sociedades logran su reproducción material (agricultura, ganadería, pesca, foreste ría, extracción) e intelectual (mitos, conocimientos, ensoñaciones, ideas, percepciones, cosmovisiones). El resultado final de esta doble interacción con la naturaleza,

\footnotetext{
${ }^{8}$ La diversidad ecológica de México está dentro de las diez naciones bioculturalmente más ricas del planeta, donde se combina la topografía y un sistema climático inusualmente variados, lo cual crea un rico mosaico de condiciones ambientales y micro ambientales. Esto se traduce en que en México se encuentran todos los grandes tipos de ambientes naturales que se conocen en el planeta, sólo la India y Perú tienen en su territorio una diversidad semejante (Toledo, 1997, 111).

9 El patrimonio social son las formas tradicionales de gobernar, que incluyen lo denominado "mano vuelta," antes se contaba con un consejo de ancianos, representado por las autoridades pasadas, quienes daban su opinión y decisión para elegir al gobernador nahua, sin embargo los conflictos que se han generado internamente tienen que ver con la inserción de instituciones, sectas y partidos políticos.
} 
Flores Pacheco, M. \& Larios León, S. (2014). Aportes para el estudio del territorio: Los Nahuas de Zongolica Veracruz. Collectivus, Revista de Ciencias Sociales, 1, (1), 70-93. Julio - Diciembre. ISSN: 2382-4018

es decir, con la diversidad biológica y ecológica, es la gran variedad de culturas que han existido y aún existen en México (Toledo, 1997, p. 131) ${ }^{10}$.

Esta sabiduría ecológica ha sido estudiada por los antropólogos desde el inicio de la disciplina, pero han aumentado las investigaciones durante las últimas décadas. Dándose cada vez más estudios multidisciplinarios que consideran que hay una íntima relación entre las culturas y los conjuntos naturales y es posible lograr una clasificación de los pueblos indígenas desde una perspectiva ecológica. Podemos hablar de las culturas de la selva, donde están representados los Teenek de Veracruz y San Luis Potosí, los Totonacos de Veracruz, los Chinantecos de Oaxaca o los Lacandones de Chiapas. Las culturas del desierto están representadas, por ejemplo, por los Otomíes de Hidalgo o Kikapues de Coahuila, entre otros, las culturas de las montañas con los Nahuas de la Sierra de Zongolica, los Tarahumaras de Chihuahua o los Coras de Nayarit.

Lo anterior se complementa con la posición de la ONU (1994) y OEA (1977) al considerar que los pueblos indígenas deberían tener todo el derecho de disfrutar y controlar su patrimonio arqueológico, histórico, y cultural. En consecuencia asegurar la protección, preservación y respeto de sus lugares sagrados y poder realizar sus ceremonias en público o en privado. El patrimonio cultural y natural contiene elementos de identidad de una cultura: Identificación, pertenencia, valores, significados, relaciones, parentesco; que permite a los hombres diferenciarse de los otros. Cada comunidad se encuentra dividida en barrios, secciones, parajes (Flores, 2007, pp. 248-249).

Por lo tanto, el concepto de Patrimonio Cultural debe revisarse y retomar la noción de cultura en su más amplia aceptación. Por ejemplo, para Hall (1989) en el Lenguaje Silencioso, menciona diez tipos de actividad humana a los que el nombra Sistemas de Mensaje Primario (SMP): Interacción, asociación, subsistencia, bisexualidad, territorialidad, temporalidad, aprendizaje, juego, defensa y explotación.

10 Sobre la relación hombre- naturaleza y sabiduría ecológica, de las creencias y rituales relacionados con la concepción del mundo, lugares sagrados, derecho consuetudinario y nahualismo podemos mencionar el artículo de Jacorzynski y López Hernández (1998) La tierra sagrada de los Tzotziles un estudio sobre ecología indígena. 
Flores Pacheco, M. \& Larios León, S. (2014). Aportes para el estudio del territorio: Los Nahuas de Zongolica Veracruz. Collectivus, Revista de Ciencias Sociales, 1, (1), 70-93. Julio - Diciembre. ISSN: 2382-4018

La cultura no es una única cosa, sino una compleja serie de actividades interrelacionadas de muchas maneras, que tienen sus orígenes profundamente enraizados". Respecto a la territorialidad señala que "...es el término que se usa para describir la toma de posesión, utilización y defensa de un territorio, por parte de los organismos vivos (...) El equilibrio de la vida en el uso del espacio es uno de los más delicados de la naturaleza. La territorialidad llega a todos los rincones y entresijos de la vida (...) (Hall, 1989, p. 158).

Como podemos observar, conceptos como el de patrimonio histórico, cultural y natural y territorio son nociones que se encuentran permanentemente interrelacionadas, y fundamentan una visión mucho más amplia. Que implica, por ejemplo, el reconocimiento de los territorios ancestrales de los pueblos indígenas, así como el derecho al uso y manejo de los recursos naturales que en ellos se encuentran. Los pueblos indígenas, a través de sus organizaciones nacionales o regionales, hoy demandan de manera conjunta el reconocimiento pleno al manejo y control de su patrimonio cultural, del mismo modo que el derecho a un desarrollo socioeconómico y cultural propio.

\subsection{Proyectos turísticos y Políticas sociales}

El turismo como fenómeno cultural estimula el florecimiento de un amplio espectro de actividades artísticas y servicios culturales, la edificación de instalaciones para el esparcimiento y la recreación. El intercambio entre personas provenientes de culturas diversas es fuente de enriquecimiento mutuo. Sin embargo, el desarrollo del turismo ha significado también, para una multitud de comunidades locales, el deterioro de su patrimonio histórico, la pérdida y la adulteración de sus valores culturales, o su reemplazo por otros ajenos a sus usos y costumbres tradicionales.

El turismo es un fenómeno ambivalente pues entraña efectos directos e indirectos, y suele traer ventajas y desventajas. Esto es, puede aportar grandes beneficios económicos y sociales a un país, una región o una localidad. Dinamizando intercambios entre diversas ramas de actividad y favoreciendo el 
Flores Pacheco, M. \& Larios León, S. (2014). Aportes para el estudio del territorio: Los Nahuas de Zongolica Veracruz. Collectivus, Revista de Ciencias Sociales, 1, (1), 70-93. Julio - Diciembre. ISSN: 2382-4018

encuentro entre personas de diferentes características sociales, culturales y económicas.

Sin embargo, el turismo implica también efectos negativos y consecuencias no deseables, como la degradación medioambiental, la pérdida de la identidad cultural y tensiones entre turistas y la población residente.

Vemos que en la actualidad el turismo en regiones indígenas va de la mano con concepto de desarrollo sostenible, el cual ha sido analizado y debatido en el marco de varias cumbres mundiales, siendo las más importantes las de Río de Janeiro y Johannesburgo. La Cumbre de Río al referirse a los pueblos indígenas, destaca el papel fundamental que ellos desempeñan en la gestión del medio ambiente, la preservación de la diversidad biológica y el desarrollo en general, debido a sus conocimientos y prácticas tradicionales. Por ello, los Estados deben reconocer y reforzar su identidad, cultura e intereses, promoviendo su participación ${ }^{11}$.

Uno de los temas más importantes que enfrentan los estudios en antropología del turismo es la del cambio cultural que se presenta en las sociedades anfitrionas o receptoras como consecuencia de la afluencia de turistas. Cambios que van acompañados por una reorganización de la población anfitriona a lo largo de líneas étnicas, es decir, la creación de etnicidades-turísticamente orientadas. No todos los nativos de una comunidad étnica estarán comprometidos para el turismo, pero quienes si lo estén terminarán formando una comunidad adicional, una "turistizada". Esta comunidad, que se constituye y representa a sí misma a lo largo de líneas étnicas se puede llamar una "comunidad etnoturistica". No obstante, hay una etnia que se encuentra allí y la identidad étnica que se construye en el escenario también es legítima y auténtica en la medida en que el turismo también es auténtico y legítimo en esos espacios sociales. Es lo que se llama Turismo étnico, porque el querer alejarse de las apariencias y penetrar en la profundidad de la vida nativa es una persecución dejada al antropólogo y no a los turistas (Azeredo Grünewald, 2006).

${ }^{11}$ Naciones Unidas: Cumbre para la Tierra: Programa 21, Río de Janeiro, 1992. 
Flores Pacheco, M. \& Larios León, S. (2014). Aportes para el estudio del territorio: Los Nahuas de Zongolica Veracruz. Collectivus, Revista de Ciencias Sociales, 1, (1), 70-93. Julio - Diciembre. ISSN: 2382-4018

La Cumbre en Sudáfrica en 2002, reafirmó los principios y la "Agenda 21" de Río de Janeiro, emitió la "Declaración de Johannesburgo sobre el Desarrollo Sostenible". En ella se ratifica el compromiso de los países miembros de la ONU en pro del desarrollo sostenible, que abarca responsabilidades en el desarrollo económico y social, al igual que la protección medioambiental (Maldonado, 2006).

En México encontramos que los territorios indígenas son visitados por un turismo que no respeta las costumbres, los espacios, los lugares sagrados, que son parte de las rutas del turismo alternativo. El problema no sólo se presenta en el territorio de los Nahuas de las Altas Montañas, se puede mencionar, por ejemplo que en la Huasteca y el desierto de San Luis Potosí hay aproximadamente cincuenta lugares de interés turístico y dentro de ellos algunos son lugares sagrados vigentes de los pueblos indígenas Nahuas y Teenek. Tal vez un dato importante es que el turismo al profanar las ofrendas, al destruir las piedras que representan a los dioses, la eficacia simbólica se pierde. Casos concretos los encontramos en Real de Catorce, La Puerta ${ }^{12}$, el Sótano de las Golondrinas, el Sótano de las Guaguas, el Sótano del Cepillo, la Cueva del Agua, el Nacimiento de Huichihuayan, entre otros (Flores, 2007, pp. 243-244).

Sin embargo, en México el patrimonio histórico, cultural y natural de los pueblos indígenas se ha convertido en uno de los principales puntos de atracción turística para aquellos que gustan estar en contacto con la naturaleza y de la convivencia con las costumbres de los que la habitan, dejando ganancias a los empresarios, a los funcionarios del gobierno federal, desarrollando negocios.

Las empresas de turismo regularmente se sienten atraídas a territorios indígenas debido a su rica herencia cultural y natural. El problema radica en que rara vez este turismo resulta en una fuente viable de empleos y de

12 Este lugar se encuentra en Amatlán, Morelos. En 1997 con el programa PROCEDE, La Puerta quedó como parte de un solar y el ejidatario se lo rento a un extranjero que construyo unos Tipis (casas de los indios pueblo), tapando la puerta y evitando que los nahuas entren, ese lugar sagrado es la entrada a otros lugares sagrados de la comunidad, ahora los problemas internos han aumentado. 
Flores Pacheco, M. \& Larios León, S. (2014). Aportes para el estudio del territorio: Los Nahuas de Zongolica Veracruz. Collectivus, Revista de Ciencias Sociales, 1, (1), 70-93. Julio - Diciembre. ISSN: 2382-4018

desarrollo económico sustentable para las comunidades en los territorios mismos. Además, este tipo de comunidades se encuentran en peligro, pues empresas transnacionales buscan apropiarse de terrenos, que en muchas ocasiones son facilitados por "caciques" o por los mismos "titulares de bienes comunales"13.

La destrucción que trae la industria del turismo como la contaminación ambiental y los grandes problemas de gestión de desechos, desplazamientos de comunidades, abusos contra los derechos humanos, trabajo y salarios injustos, desconocimiento de las culturas, etc., han perjudicado a muchos pueblos y comunidades indígenas no solo en México sino alrededor del mundo. El turismo tiene una larga historia de colonización y relaciones desiguales entre pueblos y regiones. Por lo tanto, los efectos del turismo no pueden ser medidos solamente en términos de generación de empleo, nivel de contaminación, desarrollo de infraestructura, o pérdida de la biodiversidad. Además, hay muchos efectos sociales y culturales, que tienen graves impactos y que deben formar parte de un análisis crítico del turismo y sus efectos en las comunidades indígenas.

Según el Convenio núm. 169 de la OIT, los pueblos indígenas y sus comunidades gozan del derecho a decidir sus propias prioridades en lo que atañe al proceso de desarrollo, a proteger y preservar el medio ambiente de los territorios que habitan, y a conservar sus propios valores, creencias, costumbres e instituciones ancestrales. Para lograr un desarrollo sostenido y equitativo, los gobiernos deben fortalecer las actividades de la economía tradicional y adoptar medidas encaminadas a allanar las dificultades que experimentan al afrontar nuevas condiciones de vida y de trabajo.

En la región de Zongolica, se observan tres territorios diferenciados por la altura (altitud), que va de los 500 a los $2500 \mathrm{msnm}$, por los diversos paisajes naturales, por la relación con la naturaleza, por una evidente unidad en

${ }^{13}$ El problema es que en la región de las Altas Montañas no existe un Plan de Manejo de las áreas naturales, ni estudios de factibilidad para conocer la capacidad de carga, si las empresas han generado posibilidades adecuadas para la conservación de los lugares. Si es o no sustentable, que tanto se deteriora, cuenta con tratamiento del agua que está siendo contaminada, tiene servicios turísticos, hay participación comunitaria, ¿cómo se está dando?, entre otros, aspectos. 
Flores Pacheco, M. \& Larios León, S. (2014). Aportes para el estudio del territorio: Los Nahuas de Zongolica Veracruz. Collectivus, Revista de Ciencias Sociales, 1, (1), 70-93. Julio - Diciembre. ISSN: 2382-4018

patrones culturales y diferentes formas de producción. En donde los Nahuas se han adaptado y conocen cada uno de sus ecosistemas y han emprendido estrategias de vida en torno a la tierra. Son fronteras culturales más no étnicas, donde el intercambio se da hacia afuera de la zona y no entre las comunidades. El siguiente cuadro describe el espacio diferenciado por parte los Nahuas, que es su patrimonio cultural y natural.

\section{Cuadro 1. Uso agroforestal del territorio indígena Nahua de la Sierra de Zongolica}

\begin{tabular}{|c|c|c|c|}
\hline Zona & Municipios & Vegetación & Productos \\
\hline \multirow[t]{2}{*}{$\begin{array}{c}\text { Tierra } \\
\text { fría o alta }\end{array}$} & $\begin{array}{c}\text { Tlaquilpa. Azompa, } \\
\text { Tehuipango, Tequila, } \\
\text { Aztancinga }\end{array}$ & $\begin{array}{l}\text { Bosque caducifolio, pino, } \\
\text { encino, durazno, pera y } \\
\text { ciruela }\end{array}$ & $\begin{array}{l}\text { Calabaza, maíz, frijol, haba y } \\
\text { chicharo. }\end{array}$ \\
\hline & & & $\begin{array}{l}\text { Explotación de borrego, } \\
\text { indumentaria más tradicional. }\end{array}$ \\
\hline $\begin{array}{l}\text { Tierra } \\
\text { templada }\end{array}$ & $\begin{array}{l}\text { Los Reyes, Tequila, } \\
\text { Atlahuilco, Mixtla de } \\
\text { Altamirano }\end{array}$ & Lomeríos, desforestación & $\begin{array}{c}\text { Café. } \\
\text { Calabaza, maíz, frijol. }\end{array}$ \\
\hline \multirow[t]{2}{*}{$\begin{array}{l}\text { Tierra } \\
\text { caliente } \\
\text { o baja }\end{array}$} & $\begin{array}{c}\text { Zongolica, Coetzala, } \\
\text { Tezonapa }\end{array}$ & $\begin{array}{c}\text { Cañadas, estrechos valles, } \\
\text { nacimiento de agua, } \\
\text { oquedades y barrancas }\end{array}$ & $\begin{array}{l}\text { Caña de Azúcar, milpas, } \\
\text { frutales y cafetales. }\end{array}$ \\
\hline & & & Calabaza, maíz, frijol. \\
\hline
\end{tabular}

Fuente: Elaboración propia.

\subsection{Los derechos indígenas}

Desde la perspectiva de los derechos indígenas, la territorialidad indígena se ha visto afectada por el impulso a proyectos de desarrollo económico bajo formas de turismo alternativo, turismo cultural, turismo comunitario, entre otros, en los que se ha ido valorando los recursos físicos, naturales, sociales y culturales de los pueblos indígenas. Pero en estos proyectos, ¿Qué papel se le ha asignado a la cultura y al territorio como patrimonio cultural de las comunidades?

La territorialidad étnica, es uno de los temas prioritarios en la plataforma de reivindicaciones indígenas, no únicamente en su condición de derecho colectivo indispensable, sino como una verdadera dimensión existencial de 
Flores Pacheco, M. \& Larios León, S. (2014). Aportes para el estudio del territorio: Los Nahuas de Zongolica Veracruz. Collectivus, Revista de Ciencias Sociales, 1, (1), 70-93. Julio - Diciembre. ISSN: 2382-4018

cada pueblo. El problema de los derechos sociales, políticos, culturales y territoriales de los pueblos indígenas en México, es que no se consideran sujetos de derecho, sino objeto de derecho (Flores, 2007, p. 234).

Los derechos indígenas incluyen una serie de temáticas que se encuentran en diferentes niveles y áreas de análisis. En el marco internacional, algunos países han reconocido que los pueblos indígenas deben ser sujetos de su propio derecho y no continuar bajo la tutela del Estado al que pertenecen. Sin embargo, a pesar de lo anterior y de existir organismos internacionales dedicados a velar por la no-violación de estos derechos territoriales, culturales, sociales, políticos, entre otros. La realidad es que los pueblos indígenas tienen muchos problemas para que se cumplan. Lo importante en este documento es reconocer el derecho a la diferencia que tienen los pueblos indígenas y en particular los pueblos originarios indígenas Nahuas de la Sierra de Zongolica.

No obstante, hay un tema que no ha sido incorporado a la legislación y que está en la reflexión y análisis de los investigadores, y es el relativo al territorio como un bien cultural y como un derecho colectivo. De hecho, junto con las demandas por autonomía y autodeterminación de los pueblos indígenas, se plantea el reconocimiento de derechos de exclusividad territorial señalando que poseen una relación intrínseca con el espacio, relación culturalmente mediada que tendría consecuencias sobre sus identidades y formas de vida. Con todo lo anterior, se ve que su aplicación se encuentra en desventaja con las sociedades posmodernas.

\section{Aportes del estudio del territorio}

La oferta de paisajes naturales y prácticas culturales para el turismo se está materializando a través de la comercialización de la naturaleza y la cultura. Al ser incorporadas a un modo particular de producción capitalista, en el cual la lógica de mercado gobierna a la dinámica ambiental y cultural.

De esta manera, el turismo fomenta lo que García Canclini (2002) denomina transnacionalización de la cultura y del capital, en el que se impone un intercambio desigual de los bienes materiales y simbólicos. Cuya finalidad 
Flores Pacheco, M. \& Larios León, S. (2014). Aportes para el estudio del territorio: Los Nahuas de Zongolica Veracruz. Collectivus, Revista de Ciencias Sociales, 1, (1), 70-93. Julio - Diciembre. ISSN: 2382-4018

es homogeneizar las distintas modalidades de producción cultural derivadas del sistema capitalista, donde no hay un reparto equitativo de los recursos, sino una ilusión de que todos pueden disfrutar, efectiva o virtualmente, de la superioridad de la cultura dominante.

En este sentido, los pueblos indígenas están siendo valorados recientemente por el mercado turístico como recursos. La cultura, aprovechada por el capital turístico, se homologa a la naturaleza: Un espectáculo, donde se miran del mismo modo las playas y las danzas indígenas.

El problema que se observa, es que estos destinos identificados con las culturas indígenas son apropiados por el capital turístico, lo que está teniendo como consecuencia la conversión de los territorios pertenecientes a los pueblos indígenas a territorios turísticos. Desarticulando las economías tradicionales (agricultura tradicional), a partir de la expansión de un nuevo uso de la tierra (como bien turístico) provocando su especulación. Esto genera un modelo que mercantiliza el paisaje y la cultura sin que la población local sea partícipe estratégica en la planificación y desarrollo del turismo, con una participación pasiva, quedando relegada a ser un mero atractivo, un recurso turístico vivo, una imagen fotográfica.

La afectación a los territorios de los pueblos indígenas, ya sea por la actividad turística o por otros proyectos, son un tema amplio y complejo. No obstante, el impacto de los proyectos en los territorios étnicos es visto por el Estado desde la perspectiva del desarrollo económico y no como un factor de desintegración cultural y social o de conservación del patrimonio natural y cultural de los pueblos indígenas. La propuesta de la investigación es retomar los diferentes conceptos que están vinculados al tema del territorio, porque se considera que es la única forma de entender la complejidad del mismo.

Todo estudio sobre territorio tendría que:

1.- Confrontar con el proceso histórico que ocupa el territorio, en términos de la apropiación de la tierra, de su ocupación y de las prácticas culturales que allí se desarrollan. 
Flores Pacheco, M. \& Larios León, S. (2014). Aportes para el estudio del territorio: Los Nahuas de Zongolica Veracruz. Collectivus, Revista de Ciencias Sociales, 1, (1), 70-93. Julio - Diciembre. ISSN: 2382-4018

2.- Evitar que parte de la cultura se utilice como recursos turísticos; ya que en el caso de los Nahuas de Zongolica, son los Xochitlalis (ceremoniales en torno al proceso del maíz y petición de lluvias), utilizando los símbolos inmersos en un sistema de creencias, ocupando las cuevas, los cerros, las cañadas, y los rituales que en ellos se practican. Elementos que se desarrollan en la vida privada de los pueblos y que en este momento se exhiben públicamente como turismo perdiendo el valor cultural de los nahuas (Coetzala y Totomachapa).

3.- Considerar que es importante que los pueblos Nahuas puedan hacer suyas las políticas públicas y hacer valer sus derechos sociales, políticos y culturales. Porque con las políticas turísticas, hay una pérdida del patrimonio histórico, cultural y natural. Sin dejar de lado la identidad.

\section{Referencias bibliográficas}

Aguirre, G. (1986). Zongolica: Encuentro de dioses y santos patronos. México: Universidad Veracruzana.

Azeredo, R. (2006). Tourism and ethnicity, Horiz.antropol. Vol. 1. Porto Alegre. Recuperado el 20 de marzo de 2013, [en línea] http://socialsciences.scielo.org/pdf/s_ha/v1nse/scs_a01.pdf

Barabas, A. M. (2004). La territorialidad simbólica y los derechos territoriales indígenas: Reflexiones para el Estado Pluriétnico. Alteridades, 14 (027), 105119.

Flores, M. y Tostado, M. (1991). Historias antiguas del Nayar. Tesis de Licenciatura en Etnología, Escuela Nacional de Antropología e Historia.

Flores, M. (2007). La Territorialidad de los pueblos indígenas nahuas y teenek de la Huasteca potosina: historia, etnografía mítica y lugares sagrados. Tesis de Doctorado en Ciencias Antropológicas, México, Universidad Autónoma Metropolitana, Iztapalapa, D.F.

Flores, M. (2010). Estudios etnográficos del área de cobertura de los Centros Coordinadores para el Desarrollo Indígena de Guachochi, Zacapoaxtla, Peto, 
Flores Pacheco, M. \& Larios León, S. (2014). Aportes para el estudio del territorio: Los Nahuas de Zongolica Veracruz. Collectivus, Revista de Ciencias Sociales, 1, (1), 70-93. Julio - Diciembre. ISSN: 2382-4018

Tlacoapa y Tlaxiaco (5 Grupos Etnolingüísticos). México: Los Tlapanecos de Guerrero, CIESAS.

García, N. (2002). Culturas populares en el capitalismo. México: Grijalbo.

Giménez, G. (2005). Territorio e identidad. Breve introducción a la geografía cultural. Revista Trayectorias, 7 (17), 8-24.

Giménez, G. \& Héau, L. C. (2007). El desierto como territorio, paisaje y referente de identidad. Culturales, 3. Recuperado el 12 de marzo de 2013, [en línea] http://www.redalyc.org/articulo.oa?id=69430502

Hall, E. T. (1989). El Lenguaje silencioso. Madrid: Alianza editorial.

López Austín. A. (1994). Tamoachan y Tlalocan. México: FCE.

Nates Cruz, B. (2011). Soportes teóricos y etnográficos sobre conceptos de territorio. Co-herencia, 8 (14), 209-229. Recuperado el 11 de mayo de 2013, [en línea] http://www.redalyc.org/articulo.oa?id=77420067009.

Maldonado, C. (2006). Turismo y comunidades indígenas: Impactos, pautas para autoevaluación y códigos de conducta. Ginebra. OIT.

Rodríguez, M. (2003) Ritual, identidades y procesos étnicos en la Sierra de Zongolica, Veracruz. México: CIESAS.

Organización de los Estados Americanos OEA. (1977). Proyecto de Declaración Americana sobre los Derechos de los Pueblos Indígenas. Recuperado el 14 de marzo [en línea] http://www.oas.org/es/cidh/indigenas/actividades/declaracion.asp

Organización de las Naciones Unidas ONU. (1994). Proyecto De Declaración sobre los Derechos de los Pueblos Indígenas. Recuperado el 16 de marzo de 2013 [en línea] http://www.un.org/es/globalissues/indigenous/ 
Flores Pacheco, M. \& Larios León, S. (2014). Aportes para el estudio del territorio: Los Nahuas de Zongolica Veracruz. Collectivus, Revista de Ciencias Sociales, 1, (1), 70-93. Julio - Diciembre. ISSN: 2382-4018

Toledo, V. (1997). La diversidad ecológica de México. En Florescano, E. (Coordinador). El patrimonio Nacional de México (11-138). México: FCECNCA.

Velasco, T. (2002). El péndulo de la resistencia: (La defensa de la territorialidad y la autonomía indígena), Gobierno del Estado de Veracruz: LIX H. Legislatura del Estado. 